\title{
A randomized, open-label, single-visit, crossover study simulating triple-drug delivery with Ellipta compared with dual inhaler combinations in patients with COPD
}

This article was published in the following Dove Press journal: International Journal of COPD

Job van der Palen ${ }^{1,2}$

Wendy Moeskops-van

Beurden'

Carolyn M Dawson ${ }^{3}$

Wai-Yee James ${ }^{3}$

Andrew Preece ${ }^{4}$

Dawn Midwinter ${ }^{4}$

Neil Barnes ${ }^{5}$

Raj Sharma ${ }^{6}$

'Department of Pulmonology, Medisch Spectrum Twente, Enschede, the Netherlands; ${ }^{2}$ Department of Research Methodology, Measurement, and Data Analysis, University of Twente, Enschede, the Netherlands; ${ }^{3}$ William Harvey Heart Centre, St Bartholomew Hospital, London, UK; ${ }^{4}$ Respiratory Therapy Area Unit, GlaxoSmithKline, Stockley Park, UK; ${ }^{5}$ Medical Department, GlaxoSmithKline, Brentford, UK; ${ }^{6}$ Respiratory Medical Franchise, GlaxoSmithKline, Brentford, UK
Correspondence: Job van der Palen Department of Pulmonary Medicine, Medisch Spectrum Twente, Koningsplein I, 75I2KZ Enschede, the Netherlands

Tel +3 I 63 I762463

Email j.vanderpalen@mst.nl
Background: Administering maintenance COPD therapy with a combination of multiple inhalers may increase inhaler errors. This study evaluated the potential benefits of using a single Ellipta dry powder inhaler (DPI) compared with two combinations of DPIs commonly used to deliver triple maintenance therapy.

Methods: Patients receiving inhaled COPD medication were enrolled in this multicenter, randomized, open-label, placebo-device, crossover study with a $2 \times 2$ complete block design (NCT0298218), which comprised two substudies: Ellipta vs Diskus + HandiHaler (substudy 1) or Turbuhaler + HandiHaler (substudy 2). Patients demonstrated inhaler use after reading the relevant patient information leaflet (PIL). A trained investigator assessed user errors (critical errors [errors likely to result in no or significantly reduced medication being inhaled] and overall errors). The primary endpoint was the proportion of patients making $\geq 1$ critical error after reading the PIL. The secondary endpoints included error rates during $\leq 2$ reassessments following investigator instruction (if required), instruction time, and patient preference.

Results: After reading the PIL, significantly fewer patients made critical errors with Ellipta compared with Diskus + HandiHaler (9\% [7/80] vs 75\% [60/80], respectively; $P<0.001)$ or Turbuhaler + HandiHaler $(9 \%[7 / 79]$ vs $73 \%$ [58/79], respectively; $P<0.001)$. The number of patients making overall errors was also lower with Ellipta vs tested inhaler combinations $(P<0.001$ for each substudy). The median instruction time needed for error-free use was shorter with Ellipta in substudies 1 and 2 (2.7 and 2.6 minutes, respectively) vs either combination (10.6 [Diskus + HandiHaler] and 11.3 minutes [Turbuhaler + HandiHaler], respectively). Significantly more patients preferred Ellipta over Diskus + HandiHaler or Turbuhaler + HandiHaler overall for taking their COPD medication ( $81 \%$ vs $9 \%$ and $84 \%$ vs $4 \%$, respectively) and per the number of steps for taking their COPD medication ( $89 \%$ vs $8 \%$ and $91 \%$ vs $5 \%$, respectively).

Conclusion: Fewer patients with COPD made critical errors with the single DPI, and patients required less instruction time, compared with each dual DPI combination.

Keywords: COPD, triple therapy, instruction, patient preference, inhaler technique, critical errors

\section{Introduction}

COPD is a growing cause of morbidity and mortality worldwide due to continued exposure to risk factors and an aging population. ${ }^{1}$ Although a chronic condition, COPD is both preventable and treatable; maintenance pharmacologic therapy is a key component of COPD management, helping to reduce symptoms and the occurrence and severity of 
exacerbations, and to improve the health status and exercise tolerance. ${ }^{2}$ Most maintenance therapies for COPD are bronchodilators (antimuscarinic drugs, $\beta 2$-agonists, methylxanthines) or anti-inflammatory agents (corticosteroids), and are administered via an inhaler device. Fixed-dose combinations with two inhaled medications in a single inhaler are widely used, and triple therapy with inhaled corticosteroid (ICS)/ long-acting $\beta$-agonist (LABA) and long-acting muscarinic antagonist (LAMA) in a single inhaler is becoming increasingly common. This may provide improved adherence to therapy compared with the use of multiple inhalers.

Instructing patients on correct inhaler use is considered an essential component of inhaled therapy. However, while instructions should be given, studies have shown that a substantial proportion of patients make $\geq 1$ critical error during inhaler use. ${ }^{3-6}$ Errors in inhalation technique are then associated with worse disease outcomes. ${ }^{7,8}$ Concerns relating to suboptimal treatment with inhaled therapies have resulted in an increased emphasis on patient training and ongoing assessment and evaluation by a trained professional. ${ }^{2}$

Regimens that depend on the use of multiple inhalers can increase the potential for inhaler errors, and thereby reduce or preclude drug delivery to the lungs. ${ }^{9-11}$ The development of fixed-dose combination inhalers that deliver multiple medications in a single inhaler has the potential to simplify treatment. This could help reduce errors and improve treatment adherence, thus leading to better outcomes., ${ }^{2,11-13}$ The Ellipta dry powder inhaler (DPI) is designed for the delivery of fixed-dose combinations of inhaled asthma and COPD medications. These include licensed combinations of a LABA and an ICS (ICS/LABA), a LAMA and a LABA (LAMA/LABA), and triple ICS/LAMA/LABA therapy (single fixed-dose inhaler triple therapy). ${ }^{14}$ In a previous study, patients made fewer overall and critical errors with the Ellipta device compared with five commonly used types of inhaler. ${ }^{15}$ Furthermore, patients showed a preference for the Ellipta device based on time needed for instruction, ease of use, and comfort of the mouthpiece. ${ }^{15}$

The aim of the current study was to evaluate the potential benefits of the single Ellipta DPI and combinations of multiple DPIs (Diskus + HandiHaler and Turbuhaler + HandiHaler) commonly used to deliver inhaled triple therapy.

\section{Materials and methods}

\section{Study design and eligibility criteria}

This was a randomized, multicenter, open-label, placebodevice, crossover study with a $2 \times 2$ complete block design and was conducted in five centers in the UK and the Netherlands (ClinicalTrials.gov identifier NCT02982187;
GlaxoSmithKline [GSK] study 206215). Patients were $\geq 40$ years old and current or former smokers with $\geq 10$ pack-years of smoking history, with a documented history of COPD according to the European Respiratory Society/ American Thoracic Society criteria. ${ }^{16}$ Eligible patients were receiving maintenance therapy with a fixed-dose ICS/ LABA combination inhaler either with or without concurrent LAMA therapy during the preceding 4 weeks. Short-acting rescue medications were also permitted. The study excluded patients with a current diagnosis of asthma and patients with recent experience (within 2 years) of the Ellipta inhaler, any capsule inhaler, the Diskus inhaler (patients in substudy 1), or the Turbuhaler (patients in substudy 2). Data on historical inhaler use beyond 2 years were not collected. The study also excluded patients with a history of alcohol or drug abuse that could interfere with the completion of the study, allergy to any component of the inhaler, or receipt of any investigational drug or medical device within 30 days of study entry or within five drug half-lives of the investigational drug (whichever was longer). Patients who were not able to read and/or complete a questionnaire were also excluded from the study.

All patients attended a study center where they underwent an initial screening assessment. A medical history was taken and inclusion/exclusion criteria were recorded. Patients in substudy 1 received the Ellipta DPI and the combination of Diskus + HandiHaler DPIs, and patients in substudy 2 received the Ellipta DPI and the combination of Turbuhaler + HandiHaler DPIs. All inhalers were unbranded and contained placebo only. Random allocation of treatment sequence and posttreatment questionnaire sequence was made using an interactive web response system. All screening, treatments, and assessments took place on the same 1-day visit.

The study was conducted in accordance with the International Conference on Harmonisation Good Clinical Practice guidelines and the 2008 version of the Declaration of Helsinki. All patients provided written informed consent before participation. The ethics and review boards of the participating institutions approved the protocol before commencement of the study. In the Netherlands, the protocol was approved by the Medical Ethical Committee Twente; this approval was valid for all study sites in the Netherlands. In the UK, the protocol was approved by the National Research Ethics Service Committee East Midlands and was valid for the UK sites.

\section{Outcomes and assessments}

For each inhaler provided, patients were asked to read the patient information leaflet (PIL). Each patient then used the inhaler while being observed by a study health care provider 
(HCP) who had been trained in the use of all the inhalers. Any patient errors in inhaler use were recorded by the HCP. If there were errors, the HCP provided instructions on correcting the observed errors and the patient attempted correct inhaler use again. The process could be repeated once more if the second attempt was unsuccessful, but no more than three attempts for the correct use were permitted. Thus, every patient read the PIL initially and could later receive one or two instructions focused on correcting errors. The HCPs used a protocol-defined checklist of inhaler errors appropriate to each inhaler type, which were categorized as overall errors or critical errors (Table 1). These checklists were based upon a review of the PIL for each inhaler and the steps defined

Table I Error checklist provided for assessment of inhaler use Ellipta

- Failed to open cover ${ }^{\mathrm{a}}$

- Shook the device after dose preparation ${ }^{a}$

- No exhalation before an inhalation

- Exhaled directly into mouthpiece ${ }^{a}$

- No seal by the lips round the mouthpiece during the inhalation ${ }^{a}$

- Inhalation maneuver was not:

I. Long

2. Steady

3. Deep

- Blocked air inlet during inhalation maneuver

- Did not hold breath

- Did not close the device (note: this is an error, but one which does not affect the medication that is inhaled)

- Any other comments: (free text box)

Diskus

- Failed to open cover ${ }^{\mathrm{a}}$

- Lever is not pushed back ${ }^{\mathrm{a}}$

- Shook the device after dose preparation ${ }^{a}$

- No exhalation before an inhalation

- Exhaled directly into mouthpiece ${ }^{a}$

- No seal by the lips round the mouthpiece during inhalation ${ }^{a}$

- Inhalation maneuver was not: I. Steady

2. Deep

- Did not hold breath

- Did not close the device (note: this is an error, but one which does not affect the medication that is inhaled)

- Any other comments: (free text box)

HandiHaler

- Failed to remove capsule ${ }^{a}$

- Failed to insert capsule into the chamber

- Did not completely close device capsule chamber (heard click when satisfactory)

- Did not pierce the capsule (HCP should check capsule was pierced) ${ }^{a}$

- Shook the device after dose preparation

- No exhalation before an inhalation

- Exhaled directly into mouthpiece ${ }^{a}$

- No seal by the lips round the mouthpiece during the inhalation ${ }^{2}$

(Continued)
Table I (Continued)

\section{HandiHaler}

- Inhalation maneuver was not: I. Slow

2. Deep

- Capsule did not rattle ${ }^{a}$

- Blocked air inlet during inhalation maneuver

- Did not hold breath

- Did not check inside the capsule chamber if powder was left/did not make a second inhalation

- Any other comments: (free text box)

Turbuhaler

- Failed to remove capa

- Did not hold device upright $\left( \pm 45^{\circ}\right.$ from vertical) during dose preparation $^{\mathrm{a}}$

- Base not twisted fully backward and forward, no click heard ${ }^{a}$

- Device tipped downward after dose preparation

- Shook the device after dose preparation ${ }^{a}$

- No exhalation before an inhalation

- Exhaled directly into mouthpiece ${ }^{\mathrm{a}}$

- No seal by the lips round the mouthpiece during the inhalation ${ }^{a}$

- Inhalation maneuver was not:

I. Forceful

2. Deep

Note to HCP: it is important that the inhalation is forceful and deep from the start for this inhaler

- Blocked air inlet during inhalation maneuver

- Did not hold breath

- Did not close the device (note: this is an error, but one which does not affect the medication that is inhaled)

- Any other comments: (free text box)

Note: a Defined as a critical error.

Abbreviation: HCP, health care provider.

for correct use, on a review of the available literature, and on a review of these errors with a group of external inhaler experts. ${ }^{15}$ Critical errors in our study were defined as errors likely to result in no or significantly reduced medication being inhaled.

The primary endpoint was the percentage of patients making $\geq 1$ critical inhaler error after reading the PIL. Any single critical error with Diskus + HandiHaler or Turbuhaler + HandiHaler was counted as a critical error for the dual-inhaler treatment. The secondary endpoints included critical errors at other time points, overall errors at each time point, time taken to demonstrate correct use of an inhaler, and inhaler preference. Inhaler preference was evaluated using a questionnaire after all inhalers had been demonstrated. To avoid response bias, the sequence in which each inhaler was mentioned in the questionnaire was randomized. Patients were asked about their preference: "which treatment do you prefer based on the number of steps needed to take your COPD medication?" and "which treatment do you prefer for taking your COPD medication?". Safety was monitored over the course of the 1-day study visit. 


\section{Statistical analysis}

Sample sizes for the study were calculated from simulations using conditional logistic regression and a two-sided 5\% significance level, applying the rates of inhaler critical errors reported previously. ${ }^{15} \mathrm{~A}$ total of 72 patients in each substudy would provide $\geq 90 \%$ power to show a statistically significant difference between the critical error rate of each of the paired treatment options. The efficacy analyses for each substudy were performed using the intent-to-treat population, defined as all patients who were randomized and who had $\geq 1$ error assessment. The primary endpoint of percentage of patients making $\geq 1$ critical error after reading the PIL, and the secondary endpoints of percentage of patients making $\geq 1$ critical error after the first and second instructions from the HCP, percentage of patients making $\geq 1$ overall error after reading the PIL, and percentage of patients making $\geq 1$ overall error after the first and second instructions from the HCP were analyzed using exact logistic regression with patient as the fixed strata and treatment option and period as the fixed effects. The method of conditional logistic regression analysis provided in the protocol was modified following database freeze and reporting and analysis plan finalization as the model did not converge due to data distribution (zero cell counts). The number of instructions from the HCP $(0,1$, or 2$)$ needed to demonstrate correct inhaler use was analyzed using the Wilcoxon signed-rank test, and the total amount of time taken to demonstrate correct inhaler use was analyzed using Kaplan-Meier methods. Treatment preference was analyzed using a Cochran-Mantel-Haenszel test, adjusted for inhaler sequence.

\section{Results}

\section{Baseline characteristics}

From 30 December 2016 through 19 June 2017, 80 and 79 patients were randomized to substudies 1 and 2 , respectively, and had $\geq 1$ subsequent error assessment. One patient in substudy 2 withdrew consent prior to the study visit. Baseline patient characteristics were similar between substudies; mean age was 65 (SD 8.6) years, and 52\% were male. Approximately one-third (33\%) had a $\geq 10$-year history of COPD (Table 2). All but two subjects were receiving fixeddose ICS/LABA at study entry (99\%); $40 \%$ of patients were receiving LAMA from a device other than HandiHaler.

\section{Efficacy}

In both substudies, significantly fewer patients using the Ellipta inhaler made $\geq 1$ critical error after reading the PIL compared with patients using Diskus + HandiHaler or Turbuhaler + HandiHaler (Table 3; Figure 1). In each substudy, $9 \%$ of patients made $\geq 1$ critical error using Ellipta compared with $75 \%$ using Diskus + HandHaler in substudy $1(P<0.001)$ and $73 \%$ of patients using Turbuhaler + HandiHaler in substudy $2(P<0.001)$. Patients made more critical and overall errors with HandiHaler than with any other inhaler (Table 3). Among the 19 patients demonstrating any error with Ellipta, the most common critical error made was exhaling directly into the mouthpiece ( $26.3 \%$ in substudy $1 ; 41.2 \%$ in substudy 2 ), as shown in Table 4. Among the 44 patients demonstrating any error with Diskus, the most common critical errors made were lever not pushed back (61.4\%), exhaling directly into mouthpiece (15.9\%), and failing to open cover (11.4\%; Table 4). Among the 45 patients demonstrating any error with Turbuhaler, the most common critical errors were base not twisted fully backward and forward (60.0\%), did not hold device upright during dose preparation $(31.1 \%)$, and exhaled directly into mouthpiece (11.1\%; Table 4). Among the 57 and 50 patients demonstrating any error with Handihaler in substudies 1 and 2, respectively, the most common critical errors were capsule did not rattle $(75.4 \%$ and $66.0 \%)$, did not completely close device capsule chamber

Table 2 Patient demographics and clinical characteristics (ITT population)

\begin{tabular}{|c|c|c|c|}
\hline Characteristic & $\begin{array}{l}\text { Substudy I: Ellipta vs } \\
\text { Diskus + HandiHaler } \\
(n=80)\end{array}$ & $\begin{array}{l}\text { Substudy 2: Ellipta } \\
\text { vs Turbuhaler }+ \\
\text { HandiHaler }(n=79)\end{array}$ & $\begin{array}{l}\text { Total } \\
(\mathbf{N}=\mid 59)\end{array}$ \\
\hline Mean (SD) age, years & $64.3(8.69)$ & $65.7(8.49)$ & $65.0(8.60)$ \\
\hline \multicolumn{4}{|l|}{ Sex, n (\%) } \\
\hline Male & $4 I(5 I)$ & $42(53)$ & $83(52)$ \\
\hline Mean (SD) BMI, kg/m² & $26.7(5.6 I)$ & $27.1(4.57)^{\mathrm{a}}$ & $26.9(5.11)^{\mathrm{a}}$ \\
\hline \multicolumn{4}{|l|}{ COPD history, n (\%) } \\
\hline 6 months to $<10$ years & $58(72.5)$ & $48(60.8)$ & $106(66.7)$ \\
\hline $10-<25$ years & $19(23.8)$ & $29(36.7)$ & $48(30.2)$ \\
\hline$\geq 25$ years & $3(3.8)$ & $2(2.5)$ & $5(3.1)$ \\
\hline
\end{tabular}

Note: a Substudy 2: $\mathrm{n}=78$ and total $\mathrm{N}=158$ due to missing BMI data for one patient.

Abbreviations: BMI, body mass index; ITT, intent to treat. 
Table 3 Number of patients with $\geq$ I critical error or overall error using Ellipta vs comparator inhalers after reading the patient information leaflet (ITT population)

\begin{tabular}{|c|c|c|c|c|}
\hline \multirow[t]{2}{*}{ Error type } & \multicolumn{2}{|c|}{$\begin{array}{l}\text { Substudy I: Ellipta vs } \\
\text { Diskus + HandiHaler }(n=80)\end{array}$} & \multicolumn{2}{|c|}{$\begin{array}{l}\text { Substudy 2: Ellipta vs } \\
\text { Turbuhaler + HandiHaler }(n=79)\end{array}$} \\
\hline & Ellipta & Diskus + HandiHaler & Ellipta & Turbuhaler + HandiHaler \\
\hline Critical errors, n (\%) & $7(9)$ & $60(75)$ & $7(9)$ & $58(73)$ \\
\hline Overall errors, $\mathrm{n}(\%)$ & $19(24)$ & $64(80)$ & $17(22)$ & $63(80)$ \\
\hline
\end{tabular}

Note: $P<0.00$ I for all comparator inhaler combinations vs Ellipta.

Abbreviation: ITT, intent to treat.

(29.8\% and $32.0 \%$ ), did not pierce the capsule $(31.6 \%$ and $28.0 \%$ ), failed to remove capsule (12.3\% and $14.0 \%)$, failed to insert the capsule into the chamber $(10.5 \%$ and $12.0 \%)$, and exhaled directly into mouthpiece $(7.0 \%$ and $12.0 \%$; Table 4). Among all patients, significantly fewer patients using the Ellipta inhaler had $\geq 1$ overall (critical and non-critical) error in the first attempt after reading the PIL, compared with patients using other inhalers $(24 \%$ vs $80 \%$ with Diskus + HandiHaler $[P<0.001]$ and $22 \%$ vs $80 \%$ $[P<0.001]$ with Turbuhaler + HandiHaler; Table 3).

\section{Instruction time}

The majority of patients required no additional instructions, other than reading the PIL, to demonstrate correct use of
Ellipta ( $76 \%$ and $78 \%$, respectively, in substudies 1 and 2 ). The majority of patients receiving Diskus + HandiHaler (substudy 1) or Turbuhaler + HandiHaler (substudy 2) required instructions from an HCP on one $(65 \%$ and $59 \%$, respectively) or two ( $8 \%$ and $14 \%$, respectively) occasions before demonstrating correct use. In both substudies, fewer patients using Ellipta ( $4 \%$ and $1 \%$, respectively) failed to demonstrate correct use after the third attempt (second instruction), compared with those using Diskus + HandiHaler (8\%) and Turbuhaler + HandiHaler $(6 \%)$. Overall, less time was required to train patients (reading the PIL and HCP instruction) on the correct way to use the Ellipta compared with Diskus + HandiHaler and Turbuhaler + HandiHaler (median of 2.7 and 2.6 vs 10.6 and 11.3 minutes, respectively). In both

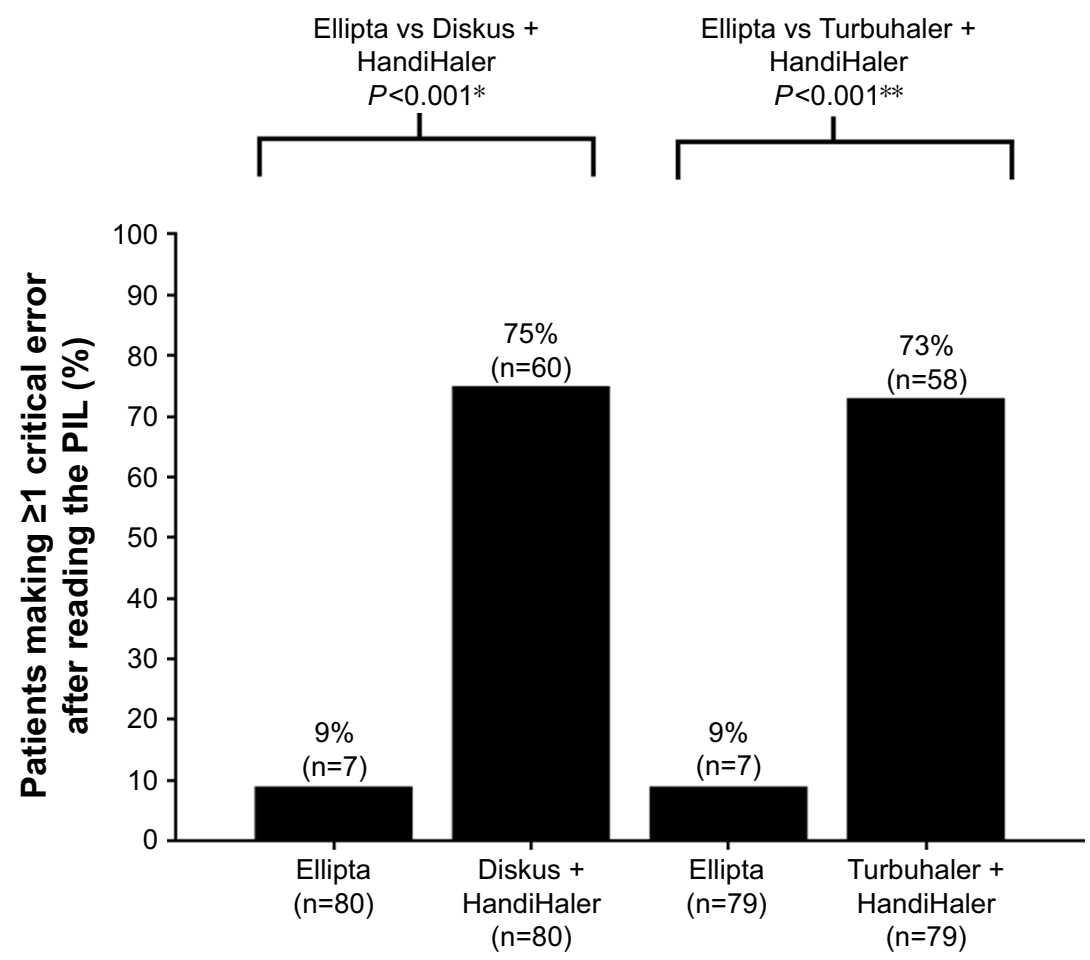

Figure I Proportion of patients with at least one critical inhaler error in substudy I (Ellipta vs Diskus + HandiHaler) and substudy 2 (Ellipta vs Turbuhaler + HandiHaler) after reading the PIL (ITT population).

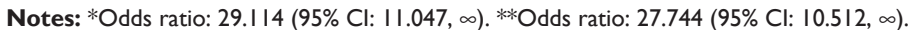

Abbreviations: ITT, intent to treat; PIL, patient information leaflet. 
Table 4 Numbers of critical use errors at first use after reading the patient information leaflet (ITT population) in substudies I and 2

\begin{tabular}{ll}
\hline $\begin{array}{l}\text { Substudy I: Ellipta vs } \\
\text { Diskus + HandiHaler (n=80) }\end{array}$ & $\begin{array}{l}\text { Number (\%) } \\
\text { of subjects }\end{array}$ \\
\hline Errors on Ellipta & \\
Any error & 19 \\
Any critical error & 7 \\
Exhaled directly into mouthpiece & $5(26)$ \\
Failed to open cover & $2(11)$ \\
No seal by the lips round the mouthpiece during & 1 I (5) \\
the inhalation & \\
Errors on Diskus & \\
Any error & 44 \\
Any critical error & 32 \\
Lever not pushed back & $27(61)$ \\
Exhaled directly into mouthpiece & $7(16)$ \\
Failed to open cover & $5(11)$ \\
Shook the inhaler after dose preparation & $1(2)$ \\
No seal by the lips round the mouthpiece during & $1(2)$ \\
the inhalation & \\
Errors on HandiHaler & \\
Any error & 57 \\
Any critical error & 54 \\
Capsule did not rattle & $43(75)$ \\
Did not pierce the capsule & $18(32)$ \\
Did not completely close inhaler capsule chamber & $17(30)$ \\
Failed to remove capsule & $7(12)$ \\
Failed to insert capsule into the chamber & $6(11)$ \\
Exhaled directly into mouthpiece & $4(7)$ \\
No seal by the lips round the mouthpiece during & $3(5)$ \\
inhalation & \\
\hline Substudy 2: Ellipta vs &
\end{tabular}

Substudy 2: Ellipta vs

Turbuhaler + HandiHaler $(n=79)$

\section{Errors on Ellipta}

Any error

Any critical error

Exhaled directly into mouthpiece

Failed to open cover

No seal by the lips round the mouthpiece during

inhalation

Shook the inhaler after dose preparation

Errors on Turbuhaler

Any error

Any critical error

Base not twisted fully backward and forward

Did not hold inhaler upright during dose preparation

Exhaled directly into mouthpiece

Failed to remove cap

No seal by the lips round the mouthpiece during

inhalation

\section{Errors on HandiHaler}

Any error

Any critical error

Capsule did not rattle

Did not completely close inhaler capsule chamber

Did not pierce the capsule

Failed to remove capsule

Failed to insert capsule into the chamber

Exhaled directly into mouthpiece

No seal by the lips round the mouthpiece during

inhalation
17

7

$7(4 I)$

$3(18)$

$2(12)$

I (6)

45

39

$27(60)$

$14(3 \mathrm{I})$

$5(\mathrm{II})$

3 (7)

I (2)

50

45

$33(66)$

$16(32)$

$14(28)$

$7(14)$

$6(12)$

$6(12)$

2 (4)
Note: ${ }^{\text {PPercentages of patients demonstrating specific critical errors were calculated }}$ as a percentage of the total number of patients who made any error on that inhaler. Abbreviation: ITT, intent to treat. substudies, the probability of patients demonstrating the correct use of the Ellipta in 5 minutes or less was $86 \%$ (Figure 2). This was notably higher than for Diskus + HandiHaler in substudy 1 and Turbuhaler + HandiHaler in substudy $2(10 \%$ and $9 \%$, respectively).

\section{Patient preference}

In both substudies, significantly more patients showed a preference for Ellipta based on the number of steps required to administer treatment ( $89 \%$ and $91 \%$ ) compared with Diskus + Handihaler and Turbuhaler + HandiHaler ( $8 \%$ and $5 \%, P<0.001$; Table 5). Patients in both substudies also demonstrated an overall preference for Ellipta for taking their COPD medication $(81 \%$ and $84 \%$ for Ellipta compared with $9 \%$ and $4 \%$ for Diskus + Handihaler and Turbuhaler + HandiHaler, respectively, $P<0.001$; Table 5). Overall, $4 \%$ of patients in both substudies did not express an inhaler preference based on the number of steps required to take medication; $10 \%$ of patients in substudy 1 and $13 \%$ of patients in substudy 2 did not express an overall inhaler preference.

\section{Safety}

Only one adverse event was reported in this single-visit study. In substudy 1, a patient experienced a laceration (cuts on both thumbs) while opening the blister card packaging of placebo Handihaler capsules.

\section{Discussion}

In this study, we evaluated the potential benefits of using single Ellipta DPI vs two combinations of DPIs commonly used to deliver triple maintenance COPD therapy. The Ellipta inhaler, designed to deliver three different medications in a single fixed dose, was found to be superior to two regimens consisting of dual inhalers. Of particular relevance, fewer than one in ten patients using Ellipta for the first time made critical errors after reading the PIL; with dual-inhaler treatments, at least seven out of every ten patients made critical errors. Critical errors result in no or significantly reduced medication being inhaled. ${ }^{17}$ Therefore, patients using Ellipta are more likely to receive the expected dose during treatment. Other studies have compared rates of errors for different inhalers in COPD and asthma. ${ }^{13,18-20}$ These were mostly observational studies and reported large differences in error rates according to inhaler type; importantly, error rates were high across all inhaler types. More recently, however, a randomized crossover study compared Ellipta with five single 


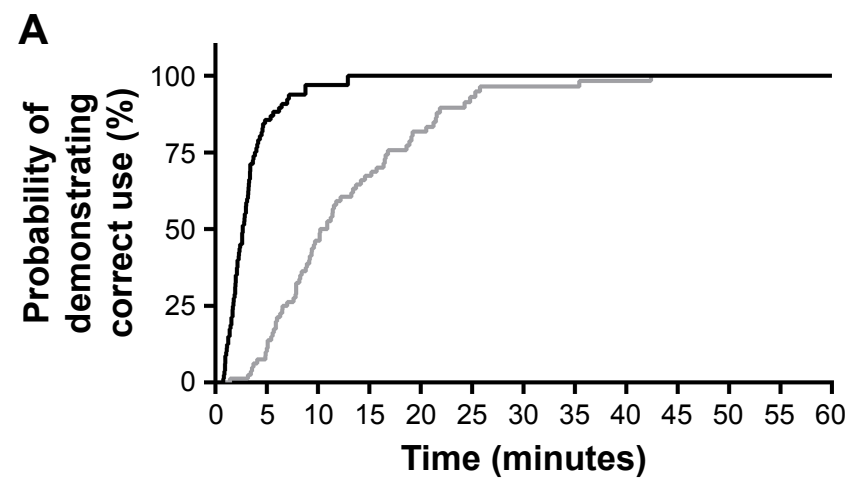

Ellipta $\longrightarrow$ Diskus + HandiHaler
B
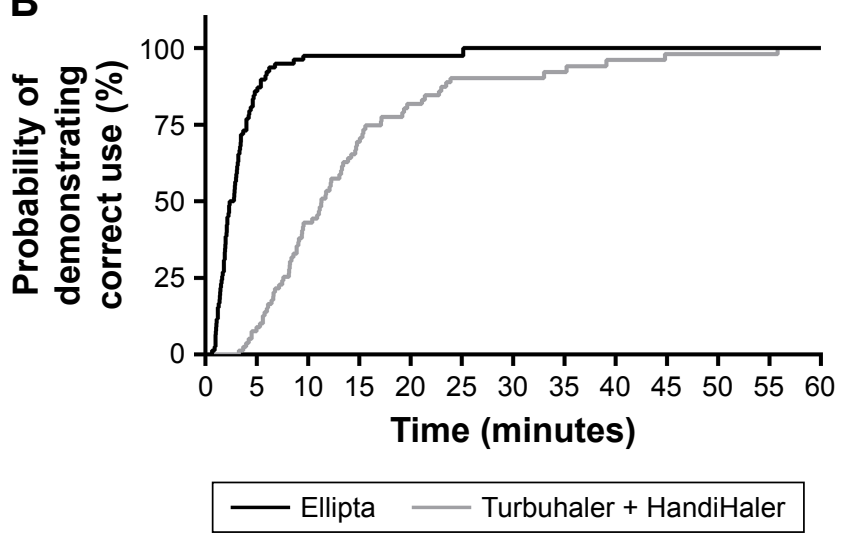

Figure 2 Kaplan-Meier plot of total time taken to demonstrate correct inhaler use.

Notes: (A) Substudy I: Ellipta vs Diskus + HandiHaler. (B) Substudy 2: Ellipta vs Turbuhaler + HandiHaler.

inhaler types (Diskus/Accuhaler, metered-dose inhaler, Turbuhaler, HandiHaler, and Breezhaler) in patients with COPD or asthma who were naïve to their respective inhalers. ${ }^{15}$ Significantly fewer COPD patients made critical errors and overall errors when using Ellipta for the first time compared with those using other inhalers. Patients also required less time to demonstrate correct use of Ellipta compared with any

Table 5 Overall inhaler preference (ITT population)

\begin{tabular}{|c|c|c|}
\hline Substudy I & $\begin{array}{l}\text { Ellipta vs } \\
\text { Diskus + HandiHaler } \\
(n=80)\end{array}$ & $P$-value \\
\hline Preference based on number & & $<0.001$ \\
\hline \multicolumn{3}{|l|}{ of steps needed to take } \\
\hline \multicolumn{3}{|l|}{ COPD medication, n (\%) } \\
\hline Ellipta & $71(89)$ & \\
\hline Diskus + HandiHaler & $6(8)$ & \\
\hline No preference & $3(4)$ & \\
\hline $\begin{array}{l}\text { Preference for taking COPD } \\
\text { medication, n (\%) }\end{array}$ & & $<0.001$ \\
\hline Ellipta & $65(8 I)$ & \\
\hline Diskus + HandiHaler & $7(9)$ & \\
\hline No preference & $8(10)$ & \\
\hline \multirow[t]{3}{*}{ Substudy 2} & Ellipta vs & \\
\hline & Turbuhaler + & \\
\hline & HandiHaler $(n=79)$ & \\
\hline Preference based on number & & $<0.001$ \\
\hline \multicolumn{3}{|l|}{ of steps needed to take } \\
\hline \multicolumn{3}{|l|}{ COPD medication, n (\%) } \\
\hline Ellipta & $72(91)$ & \\
\hline Turbuhaler + HandiHaler & $4(5)$ & \\
\hline No preference & $3(4)$ & \\
\hline $\begin{array}{l}\text { Preference for taking COPD } \\
\text { medication, n (\%) }\end{array}$ & & $<0.001$ \\
\hline Ellipta & $66(84)$ & \\
\hline Turbuhaler + HandiHaler & $3(4)$ & \\
\hline No preference & $10(13)$ & \\
\hline
\end{tabular}

Abbreviation: ITT, intent to treat. of the other inhalers. The authors concluded that the lower error rates were most likely due to the intuitive design of Ellipta, which required fewer steps to deliver the medication, and the shorter PIL. ${ }^{15}$

The amount of time patients spent in training (ie, reading the inhaler PIL or receiving instruction from an HCP) was also significantly shorter with Ellipta compared with dualinhaler treatments. The instruction and training received and the ability of a COPD patient to use the prescribed inhaler(s) correctly are important factors in ensuring effective drug delivery. ${ }^{9,13}$ In short, for any prescribed inhaler, the patient needs to follow all the steps in the PIL correctly in order to ensure optimal drug delivery. Instruction from an HCP (nurse, physician, or community pharmacist) should be given to improve patients' inhaler technique both when initially prescribed and in subsequent reinforcement training. ${ }^{21}$ However, such training is often not provided in clinical practice. Reduction in the time needed for training patients may itself reduce barriers to treatment. In a survey of pharmacists, lack of pharmacist time and patient-related factors (including patients' own time) were the main barriers to optimal asthma care. ${ }^{22}$

Patients showed a preference for Ellipta compared with either dual-inhaler therapy due to the number of steps required for use (substudy 1, 89\% of patients; substudy 2, $91 \%$ of patients) and as an overall preference for administering COPD medication ( $81 \%$ and $84 \%$, respectively), though no such medications were given in this study. This is important because greater treatment satisfaction is associated with better treatment adherence. ${ }^{23-25}$ Higher rates of treatment adherence are then associated with lower exacerbation rates in COPD..$^{24,25}$ 
Strengths of the current study include the use of placebo inhalers, which can help to eliminate bias related to effects of the medication rather than the design of the inhaler itself. By restricting the study to inhaler-naïve patients and by using a randomized crossover design, there was also reduced patient variability. As the study was conducted at selected sites with trained nurses to assess inhaler technique, variability was minimized. The study attempted to use standardized checklists that were individualized for each type of inhaler to optimize consistency between observers in identifying critical errors, but it is recognized that consensus is still required in this area.

A limitation of this study is that it only looked at the use of an inhaler over the course of a single day, so was unlikely to capture whether knowledge of the inhaler was retained over the long term. Furthermore, health literacy of patients was not assessed; however, investigators were required to recruit patients whom they deemed were able to read and understand the appropriate PIL. This study highlights the need for retention and reinforcement of training: even with Ellipta, 9\% of patients made a critical error when relying only on the PIL. As this study examined initial training with inhalers containing placebo, these data do not provide information on long-term inhaler technique and its association with clinical outcomes or on differences between inhalers in properties such as susceptibility to moisture on long-term storage. Although participants in this study were all naïve to the single fixed-dose inhaler and comparator inhalers for the previous 2 years, the design was open label and involved an element of subjective assessment on some parameters of inhaler error, despite efforts to standardize training. It is also recognized that historical use of the tested devices beyond the previous 2 years may have obscured slightly the differences in technique observed between them. Finally, a scenario in which patients are required to learn to use three inhalers during a single visit does not reflect general clinical practice and may have been a particular disadvantage in the dual-inhibitor groups.

\section{Conclusion}

Inhaler errors are more likely to occur with the use of multiple inhaler types with different techniques. Single inhaler types, such as Ellipta, offer the advantage of reducing such errors. Here, a single fixed-dose inhaler was associated with significantly fewer critical errors compared with dual inhalers in patients with moderate-to-severe COPD. The single fixeddose inhaler was also preferred by patients and required reduced training time compared with dual inhalers.

\section{Acknowledgments}

The authors would like to thank Valerie Millar (GSK, Uxbridge, UK), Rehan Ali (Lorien, UK), and Collins Mutua (Phastar, Kenya) for their support in data analysis for this paper. Editorial support (in the form of writing assistance, collating author comments, assembling tables and/or figures, grammatical editing, fact checking, and referencing) was provided by Catherine Elliott, BSc, of Gardiner-Caldwell Communications (Macclesfield, UK) and was funded by GSK. Ellipta and Diskus are trademarks owned by or licensed to the GSK group of companies. Turbuhaler is a trademark of AstraZeneca. HandiHaler is a trademark of Boehringer Ingelheim. This study was funded by GSK (ClinicalTrials. gov number NCT02982187; GSK study 206215).

\section{Author contributions}

Literature search: DM, JvdP. Study design: DM, JvdP, AP. Data collection: WMvB. All authors contributed toward data analysis and interpretation, drafting and critically revising the paper, gave final approval of the version to be published, and agree to be accountable for all aspects of the work.

\section{Disclosure}

AP, RS, DM, and NB are employees of GSK and hold stocks and/or shares. The authors report no other conflicts of interest in this work.

\section{References}

1. Mathers CD, Loncar D. Projections of global mortality and burden of disease from 2002 to 2030. PLoS Med. 2006;3(11):e442.

2. Global Initiative for Chronic Obstructive Lung Disease (GOLD). Global Strategy for the Diagnosis, Management, and Prevention of COPD (Updated 2017). Available from: https://goldcopd.org/gold-2017-globalstrategy-diagnosis-management-prevention-copd/. Accessed July 20, 2018.

3. van der Palen J, Klein JJ, Kerkhoff AH, van Herwaarden CL. Evaluation of the effectiveness of four different inhalers in patients with chronic obstructive pulmonary disease. Thorax. 1995;50(11):1183-1187.

4. Lavorini F, Magnan A, Dubus JC, et al. Effect of incorrect use of dry powder inhalers on management of patients with asthma and COPD. Respir Med. 2008;102(4):593-604.

5. Al-Showair RA, Pearson SB, Chrystyn H. The potential of a 2Tone Trainer to help patients use their metered-dose inhalers. Chest. 2007; 131(6):1776-1782.

6. van Beerendonk I, Mesters I, Mudde AN, Tan TD. Assessment of the inhalation technique in outpatients with asthma or chronic obstructive pulmonary disease using a metered-dose inhaler or dry powder device. J Asthma. 1998;35(3):273-279.

7. Molimard M, Raherison C, Lignot S, et al. Chronic obstructive pulmonary disease exacerbation and inhaler device handling: real-life assessment of 2935 patients. Eur Respir J. 2017;49(2):1601794.

8. Price DB, Román-Rodríguez M, Mcqueen RB, et al. Inhaler Errors in the CRITIKAL Study: type, frequency, and association with asthma outcomes. J Allergy Clin Immunol Pract. 2017;5(4):1071-1081.

9. Cochrane MG, Bala MV, Downs KE, Mauskopf J, Ben-Joseph RH. Inhaled corticosteroids for asthma therapy: patient compliance, devices, and inhalation technique. Chest. 2000;117(2):542-550. 
10. Bosnic-Anticevich S, Chrystyn H, Costello RW, et al. The use of multiple respiratory inhalers requiring different inhalation techniques has an adverse effect on COPD outcomes. Int J Chron Obstruct Pulmon Dis. 2017;12:59-71.

11. van der Palen J, Klein JJ, van Herwaarden CL, Zielhuis GA, Seydel ER. Multiple inhalers confuse asthma patients. Eur Respir J. 1999;14(5): 1034-1037.

12. Bonini M, Usmani OS. The importance of inhaler devices in the treatment of COPD. COPD Res Pract. 2015;1(1):9.

13. Melani AS, Bonavia M, Cilenti V, et al. Inhaler mishandling remains common in real life and is associated with reduced disease control. Respir Med. 2011;105(6):930-938.

14. Grant AC, Walker R, Hamilton M, Garrill K. The ELLIPTA ${ }^{\circledR}$ dry powder inhaler: design, functionality, in vitro dosing performance and critical task compliance by patients and caregivers. J Aerosol Med Pulm Drug Deliv. 2015;28(6):474-485.

15. van der Palen J, Thomas M, Chrystyn H, et al. A randomised openlabel cross-over study of inhaler errors, preference and time to achieve correct inhaler use in patients with COPD or asthma: comparison of ELLIPTA with other inhaler devices. NPJ Prim Care Respir Med. 2016;26:16079.

16. Celli BR, Macnee W, ATS/ERS Task Force. Standards for the diagnosis and treatment of patients with COPD: a summary of the ATS/ERS position paper. Eur Respir J. 2004;23(6):932-946.

17. Usmani OS, Lavorini F, Marshall J, et al. Critical inhaler errors in asthma and COPD: a systematic review of impact on health outcomes. Respir Res. 2018;19(1):10.
18. Hesselink AE, Penninx BW, Wijnhoven HA, Kriegsman DM, van Eijk JT. Determinants of an incorrect inhalation technique in patients with asthma or COPD. Scand J Prim Health Care. 2001;19(4):255-260.

19. Batterink J, Dahri K, Aulakh A, Rempel C. Evaluation of the use of inhaled medications by hospital inpatients with chronic obstructive pulmonary disease. Can J Hosp Pharm. 2012;65(2):111-118.

20. van der Palen J, Eijsvogel MM, Kuipers BF, Schipper M, Vermue NA. Comparison of the Diskus inhaler and the Handihaler regarding preference and ease of use. J Aerosol Med. 2007;20(1):38-44.

21. Klijn SL, Hiligsmann M, Evers S, et al. Effectiveness and success factors of educational inhaler technique interventions in asthma \& COPD patients: a systematic review. NPJ Prim Care Respir Med. 2017;27(1):24.

22. Kritikos VS, Reddel HK, Bosnic-Anticevich SZ. Pharmacists' perceptions of their role in asthma management and barriers to the provision of asthma services. Int J Pharm Pract. 2010;18(4):209-216.

23. Anderson P. Patient preference for and satisfaction with inhaler devices. Eur Respir Rev. 2005;14(96):109-116.

24. Mäkelä MJ, Backer V, Hedegaard M, Larsson K. Adherence to inhaled therapies, health outcomes and costs in patients with asthma and COPD. Respir Med. 2013;107(10):1481-1490.

25. Chrystyn H, Small M, Milligan G, et al. Impact of patients' satisfaction with their inhalers on treatment compliance and health status in COPD Respir Med. 2014;108(2):358-365.
International Journal of COPD

\section{Publish your work in this journal}

The International Journal of COPD is an international, peer-reviewed journal of therapeutics and pharmacology focusing on concise rapid reporting of clinical studies and reviews in COPD. Special focus is given to the pathophysiological processes underlying the disease, intervention programs, patient focused education, and self management protocols.

\section{Dovepress}

This journal is indexed on PubMed Central, MedLine and CAS. The manuscript management system is completely online and includes a very quick and fair peer-review system, which is all easy to use. Visit http://www.dovepress.com/testimonials.php to read real quotes from published authors. 